\section{Aspectos metodológicos de las encuestas de salud por entrevista: aportaciones de la Encuesta de Salud de Barcelona 2006}

\section{Carme Borrell ${ }^{1,2,3}$}

\section{Maica Rodríguez-Sanz ${ }^{1,3}$}

'Agencia de Salut Pública de Barcelona

${ }^{2}$ Universitat Pompeu Fabra

${ }^{3}$ Ciber de Epidemiología y Salud Pública (CIBERESP), España

\section{Resumen}

Las encuestas de salud son el procedimiento de elección para obtener información de la población de una determinada zona sobre fenómenos subjetivos relacionados con la salud tales como la existencia de síntomas actuales o pasados, limitaciones o incapacidades generadas por problemas de salud, hábitos personales, exposiciones a situaciones de riesgo.

Este artículo tiene por objetivo presentar los pasos seguidos en el diseño de una encuesta de salud y explicar algunos de los instrumentos utilizados en la Encuesta de Barcelona de 2006.

Se presentan las novedades de la Encuesta de Salud de Barcelona 2006 relacionadas con: la clase social neomarxista de EO Wright, los trabajos productivo y reproductivo, la inmigración extranjera, la discriminación percibida, la utilización de servicios sanitarios y finalmente, el cuestionario específico para menores.

Palabras-clave: encuesta de salud, métodos epidemiológicos, desigualdades en salud

Dirección de correspondencia: Carme Borrell. Agència de Salut Pública de Barcelona, Plaça Lesseps 1, 08023 Barcelona. Tel: 34-93-2384545. Fax:34-93-2173197. E-mail: cborrell@aspb.cat 


\section{Abstract}

Health interview surveys are used to study subjective health problems of the population sucg as the existence of symptoms, limitations or disabilities generated by health problems, health behaviours, exposures, risk situations, etc. The objective of this article is to present the steps followed in the design of health interview surveys and to explain some of the aspects analyzed in the 2006 Barcelona Health Survey.

It presents new aspects of the Barcelona Health Survey related to the neo-marxist social class of EO Wright, productive and reproductive labor, foreign immigration, perceived discrimination, health services utilization and, finally, health questionnaire for children.

Keywords: health survey, epidemiologic methods, inequalities in health

\section{Introducción}

Las encuestas de salud son el procedimiento de elección para obtener información de la población de una determinada zona sobre fenómenos subjetivos relacionados con la salud tales como la existencia de síntomas actuales o pasados, limitaciones o incapacidades generadas por problemas de salud, hábitos personales, exposiciones a situaciones de riesgo, etc. Habitualmente también se obtiene información sobre condiciones de vida, de la vivienda, del trabajo remunerado y no remunerado. Por lo tanto, mediante una encuesta de salud dirigida a la población se pretende obtener datos de un número suficiente de personas de un modo sistemático y estructurado para el conocimiento y la monitoritzación del estado de salud y de sus determinantes ${ }^{1,2}$.

Así pues, las encuestas de salud son un instrumento importante para recolectar información que se puede obtener directamente de la población y que no se puede obtener mediante los registros rutinarios. Permiten conocer aquellas necesidades de la población que no se transforman en demanda, además de proporcionar denominadores poblacionales útiles para el cálculo de tasas y estimaciones del número de personas a riesgo.

Los motivos para realizar una encuesta de salud son los siguientes:

- Conocer los problemas de salud percibida y las conductas relacionadas con la salud de la población.

- Conocer el patrón de utilización de servicios de salud tanto preventivos como curativos o de rehabilitación.

- Analizar la relación entre el estado de salud, las conductas relacionadas con la salud y la utilización de servicios sanitarios con los factores socioeconómicos.

- Monitorizar y evaluar indicadores sociosanitarios como, por ejemplo, la población que vive sola, indicadores de necesidad como la población con limitación de la actividad, indicadores de necesidad no atendida, equidad, etc. 
- Comparar en el espacio (en distintas zonas) o en el tiempo (si se realizan encuestas periódicamente) los distintos aspectos mencionados anteriormente.

Este artículo tiene por objetivo presentar los pasos seguidos en el diseño de una encuesta de salud y explicar algunos de los instrumentos utilizados en la Encuesta de Barcelona de 2006.

\section{El diseño de las encuestas de salud}

Los aspectos metodológicos de las encuestas de salud son importantes para garantizar la calidad de las encuestas. La calidad de los datos está determinada por la validez y la repetibilidad de las preguntas del cuestionario, es por esta razón por lo que se aconseja utilizar cuestionarios estandarizados y a ser posible que se hayan utilizado previamente. Otros factores que determinan la calidad de los datos y de las inferencias que de ellos sobrevengan son la cobertura de la población entrevistada, las no respuestas y las características del entrevistador y de la persona entrevistada. La existencia de barreras psicosociales en el curso de la entrevista se reflejan principalmente en el momento de investigar cuestiones tales como el consumo de alcohol, los hábitos sexuales, los ingresos económicos, etc. De hecho, las condiciones ideales para que un fenómeno sea declarado se cumplen cuando el entrevistado es preguntado sobre algo importante para él y sobre lo cual no tiene inconveniente en hablar.

A continuación se describen las distintas fases a tener en cuenta en el diseño de una encuesta de salud.

\section{Definición de los objetivos de la encuesta}

Antes de empezar una encuesta de salud debe quedar claro cuales son los objetivos de la misma ya que ellos nos determinarán la mayoría de aspectos metodológicos del diseño de la encuesta.
Definición de la población de estudio y del período de tiempo a recolectar información

La población de estudio es la que se pretende incluir en la encuesta y depende de los objetivos del estudio. En muchas ocasiones se suelen excluir las personas institucionalizadas (residencias de ancianos, hospitales, prisiones, etc.) debido a la dificultad de muestrearlas, pero no debe olvidarse que este tipo de población suele tener unas características distintas del resto.

Otro aspecto a tener en cuenta son los niños y niñas menores de 16 años. Habitualmente la información se obtiene de otros informantes indirectos (proxy), que suele ser la madre. Este aspecto debe ser valorado cuidadosamente al diseñar el cuestionario ya que hay algunos temas (como por ejemplo el consumo de alcohol o tabaco) que deberían ser preguntados a los jóvenes, sin la presencia de sus padres.

El período de recolección de información se refiere al período en que se va a realizar el trabajo de campo. Cuando se esperan variaciones estacionales, es necesario que el período abarque todo un año. En caso contrario, se puede realizar durante unos meses.

\section{Definición de las variables}

Debe formularse la definición conceptual y operativa de cada una de las variables que se quieren obtener. La definición conceptual es la que define el concepto (ej.: la talla es la estatura de una persona). La definición operativa es la que se va a utilizar en la encuesta (ej.: la talla es la respuesta que se obtiene con la pregunta ¿Cuánto mide usted?)

\section{Diseño del instrumento de recolección de datos (cuestionario)}

Deben incluirse todas las preguntas que sean necesarias, siendo claras y concisas y evitando preguntas redundantes ${ }^{3}$. 
Las preguntas pueden ser abiertas o cerradas (precodificadas). Las preguntas abiertas permiten que el entrevistado formule sus propias respuestas lo que posteriormente implica un esfuerzo importante de codificación. Las preguntas cerradas son más sencillas de analizar, pero requieren que previamente se haya pensado en todos los tipos de respuestas posibles, además de que éstas sean mutuamente excluyentes y no planteen dudas a la hora de escoger la respuesta.

Debe tenerse en cuenta que hay preguntas que no deben realizarse a toda la población. Así por ejemplo, las prácticas preventivas en ginecología sólo se preguntan a las mujeres. En estos casos suele ponerse un filtro o una señal que indique esta cuestión.

Otro aspecto a tener en cuenta es el sesgo de memoria, es decir, el hecho de que una persona no recuerde los eventos relacionados con su salud por un largo período de tiempo. La probabilidad de que se declare un evento depende directamente de la importancia que le de una persona e inversamente del tiempo transcurrido desde que se produjo. Por lo tanto es necesario adecuar el periodo recordatorio de las preguntas, así por ejemplo, las consultas realizadas al médico se suelen referir a los últimos 15 días antes de la entrevista y en cambio, los ingresos hospitalarios se refieren al año anterior, ya que este es un evento que se olvida más difícilmente.

\section{Recolección de datos}

Es necesario definir el método de recolección de datos que se va a utilizar:

- Encuesta de salud por entrevista: Habitualmente se realizan las entrevistas en el domicilio de los entrevistados por medio de encuestadores adiestrados. El encuestador suele ir preguntando y anotando las respuestas. En las preguntas con un número amplio de respuestas suele ser útil acompañarse de una tarjeta que muestre las posibles respuestas. Utilizando el método de entrevista, el cuestionario suele estar mejor completado. De todos modos, las partes del cuestionario más confidenciales, como por ejemplo las prácticas sexuales, deberían ser contestadas directamente por el mismo entrevistado, lo que facilitaría la confidencialidad de las respuestas.

- Cuestionarios telefónicos: Este es un método más económico de entrevistar a la gente. De todos modos, depende de la cobertura telefónica, ya que si ésta no es amplia, no se puede obtener una muestra representativa de la población. Debe tenerse en cuenta que no es un método tan idóneo para preguntar aspectos sensibles, como por ejemplo la salud mental o las prácticas sexuales.

- Cuestionarios postales: En este caso se manda por correo el cuestionario y la persona entrevistada debe completarlo y mandarlo nuevamente. También es un método barato, pero tiene el inconveniente de que suele presentar una porcentaje de no respuestas elevado. Suele ser un buen método para obtener aspectos más confidenciales de la salud de las personas.

- Exámenes de salud. Este tipo de encuestas incluyen, además de la entrevista, un examen de salud donde se realizan diferentes medidas antropométricas: toma de la tensión arterial, peso, talla, pruebas funcionales respiratorias, análisis de sangre o de orina, etc. Estas encuestas son más dificultosas de realizar y mucho más caras.

\section{La muestra: tipo de muestreo, unidad de análisis y tamaño}

Tipo de muestra: La muestra debe ser representativa de la población objetivo del estudio. Existen diferentes métodos de muestreo entre los que cabe destacar:

- El muestreo aleatorio simple: Los elementos de la muestra se eligen al azar. En la práctica, equivale en sacar al azar del censo de la población objeto del estudio, los elementos que van a for- 
mar parte de la muestra. Para esa selección al azar, se usan las tablas de números aleatorios. El muestreo aleatorio simple se aplica fundamentalmente en poblaciones pequeñas $\mathrm{y}$ plenamente identificables.

- El muestreo estratificado: Si el universo no es homogéneo, sino que está formado por estratos diferentes que constituyen categorías importantes para la investigación, la elección de la muestra no debe hacerse globalmente para todos los estratos a la vez. Así por ejemplo, si nos interesa obtener estimaciones para los distritos de una ciudad, interesará muestrear cada uno de ellos con un número suficiente de entrevistas (independientemente de la población de cada distrito), siendo en este caso, un muestreo estratificado por distrito. Para determinar los estratos se suele recurrir a variables espaciales (comunidades, provincias, municipios, distritos, por ejemplo), o subdivisiones inherentes al universo en estudio (enseñanza pública/privada, por ejemplo). El muestreo estratificado es el más utilizado en la práctica. Una vez definidos los estratos, dentro de cada uno de ellos se lleva a cabo un muestreo aleatorio simple.

- El muestreo por conglomerados: En este muestreo, las unidades muestrales no son simples, sino que son colectivos. Por ejemplo, las escuelas, los hospitales, etcétera. Cada uno de estos colectivos reciben el nombre de conglomerados. Se pretende que los conglomerados sean homogéneos entre sí, sin embargo, que las unidades que las componen sean heterogéneas. Por ejemplo, los municipios, como conglomerados son homogéneos, en cambio, dentro de ellos, hay heterogeneidad porque hay población de diferentes características. La diversidad se encuentra dentro del conglomerado. Si los conglomerados elegidos son pocos, la encuesta los incluye a todos; si son muchos, hay que elegir una muestra de conglomerados. Una vez elegidos los conglomerados, dentro de cada uno de ellos se suele llevar a cabo un muestreo aleatorio simple.

Es necesario disponer de alguna fuente de información fidedigna que permita obtener los individuos a entrevistar. Habitualmente se suele utilizar el censo, algún registro de población, registros electorales, directorios de teléfonos, etc. En el caso de que no exista ninguna fuente puede recurrirse a otros métodos como por ejemplo la selección aleatoria de viviendas y dentro de ellas, de individuos.

Unidad de análisis: Se debe definir cual va a ser la unidad de análisis a utilizar. Las dos unidades más usados son el individuo o el hogar (unidad familiar), en éste último caso se trata de entrevistar a todos los componentes de la unidad familiar, la cual es escogida de forma aleatoria.

Tamaño de la muestra: El cálculo del tamaño de la muestra a entrevistar dependerá fundamentalmente de las zonas geográficas a las que se quieren inferir los resultados obtenidos. Se puede afirmar, que cuanto más pequeña sea la zona en donde se quieran inferir los resultados mayor deberá ser el tamaño de la muestra. Así por ejemplo, es distinto si se quieren dar resultados para el conjunto de Brasil que para cada uno de los estados.

\section{Planificación del trabajo de campo.}

Debe planearse el trabajo de campo teniendo en cuenta los siguientes aspectos:

- Planificación del calendario de las entrevistas a realizar: Deben definirse los lugares y los días en que se van a realizar entrevistas.

- Selección de los entrevistadores.

- Elaboración del Manual del entrevistador, donde se expliquen todos los aspectos relacionados con la Encuesta que puedan ser interesantes 
para los encuestadores: objetivos de la misma, organismo responsable, funciones de los encuestadores, descripción del cuestionario y de cada una de las preguntas, etc.

- Adiestramiento y capacitación de los encuestadores: Se realizarán dos o tres sesiones de entrenamiento de los encuestadores, donde se les explicarán todos los temas tratados en el Manual. Asimismo se realizará un ensayo del cuestionario.

- Codificación de la información: Debe definirse cómo se codificará la información y quién será el encargado de llevarla a cabo.

- Control de calidad de la información: Es necesario supervisar la recolección de la información, el seguimiento de la muestra (debe ser representativa de la población) y el proceso de codificación. Debe definirse cómo y quién realizará estas tareas.

\section{Diseño de la prueba piloto.}

La prueba piloto se realiza con la finalidad de conocer la factibilidad de la encuesta: la facilidad o dificultad de encontrar a las personas a entrevistar, la comprensión de las preguntas del cuestionario, la duración del cuestionario, etc. Suele llevarse a cabo en un número pequeño de personas (unas 50) de distintos grupos de edad, sexo y nivel educativo y se realiza durante un período corto de tiempo (una o dos semanas).

Una vez finalizada la prueba piloto e identificadas las dificultades que conlleva la realización de la encuesta, deberán efectuarse los cambios oportunos en el cuestionario.

\section{Planificación del análisis de los datos.}

Antes de iniciar el trabajo de campo, es interesante que se planifique el análisis de los datos, diseñando las principales tablas que se pretenden obtener una vez recolectada la información. La planificación del análisis que posteriormente se querrá realizar con los datos ayuda, en muchas ocasiones, a perfeccionar y/o rectificar cuestiones previamente planteadas ya que, por ejemplo, la forma de recolectar una variable depende de cómo ésta se quiera analizar con posterioridad.

Habitualmente se suelen analizar las variables más importantes (estado de salud, utilización de servicios sanitarios, conductas relacionadas con la salud, etc.) según grupo de edad, sexo y clase social. De todos modos, la potencialidad del instrumento encuesta de salud permite analizar conjuntamente muchas más variables, así por ejemplo se podrían estudiar los factores predictivos o variables relacionadas con la vacunación.

\section{Algunos instrumentos utilizados en la Encuesta de Salud de Barcelona de 2006}

En Barcelona, la primera encuesta de salud por entrevista se realizó el año 1983 siendo la primera encuesta realizada en el Estado español. La encuesta del año 2006 es la quinta que se realiza en la ciudad. Es necesario señalar que en cada uno de los cuestionarios se han realizado cambios.

La Encuesta de Salud de Barcelona del año 2006 se realizó conjuntamente con la Encuesta de Salud de Catalunya. La mitad de la muestra proviene de la Encuesta de Salud de Catalunya y la otra mitad es una ampliación de la muestra que se hace desde la Agencia de Salut Pública de Barcelona y que contempla algunos aspectos específicos de carácter social. Algunos de los aspectos que se han tenido en cuenta en esta encuesta de salud de Barcelona del año 2006 son los que se describen a continuación.

\section{La clase social neomarxista de EO Wright}

Wright ha trabajado el concepto de posiciones de clase basadas en la teoría marxista. Considera que "las estructuras de clase designan mecanismos reales, procesos 
causales que existen independientemente del teórico" ${ }^{4}$. Basándose en Marx, postula que los mecanismos de clase generan 3 efectos: los intereses materiales, la experiencia vivida y la capacidad colectiva. Los "intereses materiales" se basan en que las relaciones sociales de producción distribuyen tanto el bienestar económico (el paquete trabajo-ocio-ingreso) como el poder económico (el control sobre el plusproducto o el producto social que queda después de que todos los factores de producción se han reproducido). El interés por mantener el bienestar económico y el poder se conectan mediante el concepto de "explotación”. Las clases sociales relacionalmente definidas no tienen simplemente diferentes intereses materiales, tienen intereses materiales antagónicos, que son la base del conflicto de clases. La "experiencia vivida" se base en la serie de prácticas que imponen las relaciones sociales de producción a las personas insertas en estas relaciones. La "capacidad colectiva" se refiere a que los miembros de una misma clase deberían tener la capacidad potencial para la acción colectiva y consecuentemente la capacidad potencial de organizar la sociedad según sus intereses.

El indicador de clase social más utilizado en los estudios empíricos sociológicos ha sido la ocupación ${ }^{5}$. El concepto de ocupación se refiere al tipo de actividad laboral realizada, es un concepto tecnológico y cultural, no de clase social; sin embargo dada su correlación con la clase, junto a su alta disponibilidad, se usa como un indicador de clase social. Es necesario diferenciar los esquemas que tratan de hacer una descripción de la estructura o posiciones de clases, de los esquemas conceptuales que intentan medir las relaciones de clase. Entre los primeros se puede incluir la Clasificación del British Registrar General y entre los segundos podemos destacar la clasificación de Wright.

En el ámbito de la salud, en el Estado Español la medida de clase social que se ha utilizado más es la propuesta por la So- ciedad Española de Epidemiología ${ }^{6}$ que se ha basado en la clasificación del British Registrar General. Esta clasificación se basa en ordenar las ocupaciones, derivando 5 clases que son: Clase I: directivos y técnicos superiores, clase II: técnicos medios, artistas y deportistas, clase III: administrativos y técnicos de apoyo, clase IV: profesiones manuales cualificadas y semicualificadas, clase V: profesiones manuales no cualificadas. Esta clasificación se ha utilizado en todas las encuesta de salud realizadas en Barcelona (además del nivel de estudios y de ingresos).

La clasificación propuesta por Wright se basa en el concepto de "intereses materiales" y concretamente en los bienes de capital (propiedad de los medios de producción), bienes de organización (nivel de autoridad en una empresa) y bienes de cualificación (titulación obtenida). A partir de estos 3 ejes propone una clasificación basada en 12 posiciones de clase en la estructura social (Figura 1). Esta propuesta se introdujo ya en la Encuesta de Salud de Barcelona del año 2000 y, de momento, ha dado lugar a diversos trabajos de investigación $\mathrm{n}^{7,8}$.

\section{Los trabajos productivo y reproductivo}

En la mayoría de sociedades industrializadas, se produce una desigual distribución en función del sexo en el mundo de la producción (económico-mercantil) y el mundo de la reproducción (ámbito doméstico-familiar, que garantiza el sostenimiento de la vida humana) que es discriminatoria para las mujeres. Ello supone que los hombres realizan mayoritariamente el trabajo productivo fuera de casa que es considerado fundamental y que es reconocido socialmente, y las mujeres básicamente se encargan de las tareas relacionadas con el cuidado de las personas, que son consideradas complementarias y sin tanto valor social. La progresiva incorporación de las mujeres al mercado laboral ha sido marcada por la existencia de una fuerte segregación labo- 


\begin{tabular}{|c|c|c|c|c|c|}
\hline Propietarios & & alariados & & \multirow[b]{2}{*}{+} & \multirow{4}{*}{$\begin{array}{l}\text { Bienes de } \\
\text { organización }\end{array}$} \\
\hline $\begin{array}{l}1 \text { Capitalistas (10 o } \\
\text { más trabajadores) }\end{array}$ & $\begin{array}{l}4 \text { Directivos } \\
\text { expertos }\end{array}$ & $\begin{array}{l}7 \text { Directivos } \\
\text { semi-expertos }\end{array}$ & $\begin{array}{l}10 \text { Directivos } \\
\text { no expertos }\end{array}$ & & \\
\hline $\begin{array}{l}2 \text { Pequeños } \\
\text { propietarios (2-9 } \\
\text { trabajadores) }\end{array}$ & $\begin{array}{l}5 \text { Supervisores } \\
\text { expertos }\end{array}$ & $\begin{array}{l}8 \text { Supervisores } \\
\text { semi-expertos }\end{array}$ & $\begin{array}{l}11 \\
\text { Supervisores } \\
\text { no expertos }\end{array}$ & 0 & \\
\hline $\begin{array}{l}3 \text { Pequeña } \\
\text { burguesía (No más } \\
\text { de } 1 \text { trabajador) }\end{array}$ & $\begin{array}{l}6 \\
\text { Trabajadores } \\
\text { expertos }\end{array}$ & $\begin{array}{l}9 \text { Trabajadores } \\
\text { semi-expertos }\end{array}$ & $\begin{array}{l}12 \\
\text { Trabajadores } \\
\text { no expertos }\end{array}$ & - & \\
\hline
\end{tabular}

Fuente: Wright $\mathrm{O}^{4}$

Figura 1 - Posiciones de clase propuestas por de Erik O. Wright

Figure 1 - Social classses as proposed by Erik O. Wright

ral según género, tanto horizontal (las mujeres se concentran en unos pocos sectores de actividad económica, sobre todo en los servicios) como vertical ya que las mujeres ocupan la mayoría de trabajos menos cualificados y con menor autoridad $^{9}$.

Desde el principio, las encuestas de salud de Barcelona han introducido variables sobre el trabajo productivo, aunque el número de preguntas se ha ido incrementando (tipo de trabajo, de jornada, de contrato, número de contratos, horas trabajadas, condiciones físicas y psicosociales del trabajo). Pero el trabajo doméstico o reproductivo (trabajo relacionado con las tareas y el cuidado de los miembros del hogar) se empezó a recoger más tarde. Así, el año 1992, en Barcelona se incluyeron variables sobre el trabajo doméstico o reproductivo de hombres y mujeres, variables que se ampliaron en las encuestas de 2000 y $2006^{10}$. Tanto el trabajo productivo como el reproductivo están relacionados con la salud y son determinantes de las desigualdades según género en salud ${ }^{11}$. La Figura 2 pone en evidencia como son mayoritariamente las mujeres las responsables de organizar las tareas del hogar (limpiar, planchar, cocinar, etc.).

\section{Nuevas realidades: la inmigración extranjera}

Cataluña es una de las 17 Comunidades Autónomas del Estado español con su propia historia, economía, política y cultura, incluyendo además su propia lengua (el catalán). Durante el siglo XX ha experimentado distintas olas de inmigraciones. En los años 60, personas de otras partes del Estado español, principalmente de las Comunidades Autónomas de Andalucía, Extremadura y Galicia vinieron a Cataluña a buscar mejores oportunidades de trabajo. Estos inmigrantes se encontraron tanto con los problemas sociales de su nueva realidad, como con la dificultad de encontrar vivienda y la discriminación en el trabajo $^{12}$. El patrón migratorio ha cambiado totalmente en los últimos años ya que actualmente la gran proporción de la población inmigrante es población extranjera, sobre todo de países no Europeos ${ }^{12,13}$. Siguiendo la misma tendencia que para el total de Catalunya, la ciudad de Barcelona recibió medio millón de inmigrantes del resto del Estado Español durante los años 50 s y 60 y en los últimos años, la inmigración extranjera ha pasado de un $1,9 \%$ de la población de la ciudad en 1995 a un 4,9\% en 2001 y un $15,9 \%$ en $2006^{14}$. 
Hombres

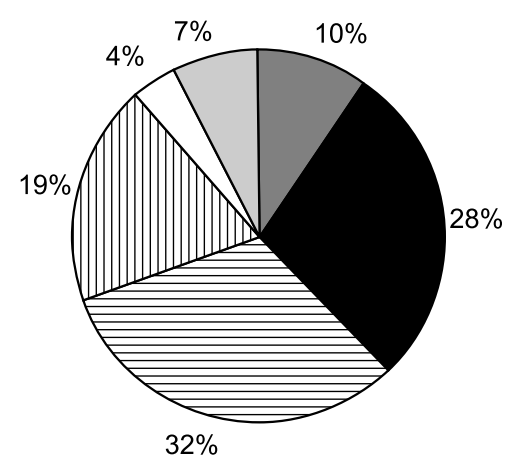

$32 \%$

$\square$ Yo solo/a

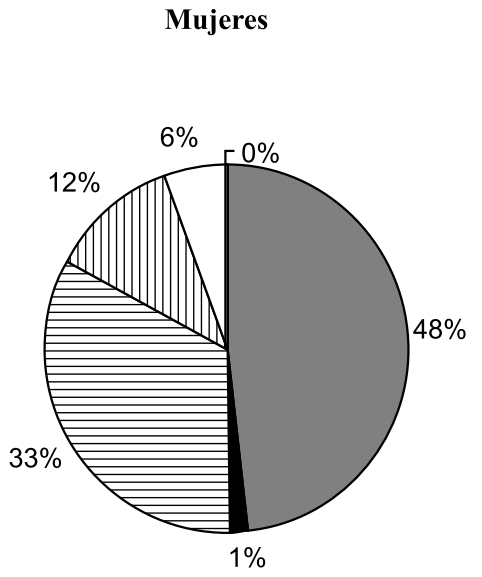

目Yo, compartiendo con mi pareja血O

$\square$ Una persona contratada

Mi pareja

Otra persona del hogar

$\square$ No consta

Figura 2 - Persona responsable de realizar las tareas del hogar según sexo. Encuesta de Salud de Barcelona-2006

Figure 2 - Person in charge of household chores per gender. Encuesta de Salud de Barcelona-2006

A partir de esta nueva realidad, el diseño de la Encuesta de Salud de Barcelona de 2006, se adaptó para poder recoger los determinantes de la salud de esta población, frecuentemente relacionados a la precariedad de sus condiciones de vida y trabajo. En este sentido se recogen preguntas relacionadas con el país de nacimiento, el año de llegada al Estado español y la discriminación percibida según etnia o sexo (ver siguiente apartado). Además, se añadieron algunas características de la vivienda que permitieran detectar condiciones precarias de vida (presencia de humedades y/o mala ventilación, insectos, humos o contaminación y ruidos).

\section{La discriminación percibida}

La discriminación se refiere a las relaciones injustas basadas en prácticas institucionales e interpersonales donde miembros de un grupo dominante adquieren privilegios a base de subordinar a otros y justifican estas prácticas mediante ideologías de superioridad o diferencias ${ }^{15}$. La discriminación puede ser legal, como por ejemplo lo fueron las leyes que impedían votar a las personas de raza negra o a las mujeres, o ilegal. Se perpetúa a través de muchos actores, tanto el estado y sus instituciones, como organizaciones privadas o bien a través de los individuos ${ }^{16}$. La discriminación afecta la salud en distintos grados, siendo el máximo nivel de expresión la violencia física y mental, como la violencia de género responsable de miles de muertes en todo el mundo ${ }^{17,18}$.

Existen pocos estudios sobre la repercusión en la salud de la discriminación tanto a nivel "macro", referido a las formas estructurales de la discriminación, como a nivel "micro" midiendo la discriminación percibida ${ }^{19}$. De todos modos, la discriminación percibida se ha relacionado con peores indicadores de salud física y percibida. En nuestro país prácticamente no hay estudios al respecto, con la excepción de uno muy reciente basado en la discriminación percibida por parte de adolescentes autóctonos e inmigrantes ${ }^{20}$. En la encuesta de salud de Barcelona se ha introducido, por primera vez, la discriminación respecto al país de origen o la etnia y respecto al sexo (Figura 3). 
Ha experimentado algún tipo de discriminación por causa de su sexo? O de su país de origen? (Se ha sentido molesto/a, se le ha negado alguna cosa o se ha sentido inferior)

\begin{tabular}{|l|l|l|l|l|}
\hline & Nunca & Algunas veces & Muchas veces & Constantemente \\
\hline $\begin{array}{l}\text { Al recibir asistencia } \\
\text { sanitaria }\end{array}$ & & & & \\
\hline Buscando trabajo & & & & \\
\hline En el trabajo & & & & \\
\hline En casa & & & & \\
\hline En un lugar público & & & & \\
\hline
\end{tabular}

Fuente: Krieger $\mathrm{N}^{19}$

Figura 3 - Pregunta sobre discriminación utilizada en la Encuesta de Salud de Barcelona-2006. Figure 3 - Question about discrimination used in the Encuesta de Salud de Barcelona-2006

Nuevas preguntas relacionadas con la utilización de servicios sanitarios

El cambio más importante realizado en la Encuesta de Salud de Barcelona 2006 respecto al uso de servicios sanitarios es la incorporación de la adaptación del instrumento Primary Care Assessment ToolsPCAT desarrollado por Starfield y Shy para conocer el grado en que la Atención Primaria de Salud (APS) de Barcelona cumple con el modelo de calidad, entendiendo este modelo como el que da respuesta a las dimensiones de la atención primaria definidas por Starfield. A partir de la versión inglesa reducida de este instrumento, se ha escogido el número de preguntas razonable a introducir en una encuesta general ${ }^{21}$. Debe señalarse que este instrumento ha sido validado también para Brasil $^{22}$.

Los instrumentos tienen como objetivo valorar la APS a partir de la medida de las siguientes características básicas definidas como propias de la APS: a) Primer contacto: la APS ha de ser el nivel de atención sanitaria con el que las personas establecen el primer contacto en caso de tener un problema de salud; b) la APS se centra en la persona y no en la enfermedad; c) globalidad de la atención; d) coordinación con el resto de niveles asistenciales. Y tres aspectos que están relacionados como son la orientación comunitaria, la centralidad en la familia y la competencia cultural.

La Figura 4 muestra el resultado encontrado para cada una de las preguntas en la ciudad de Barcelona.

\section{Cuestionario específico para menores}

Habitualmente el cuestionario dirigido a menores ha sido el mismo que el de la población adulta pero con un número inferior de preguntas. En esta última encuesta de 2006, por primera vez en Barcelona, se diseñó un cuestionario específico para los menores distinto que el de la población adulta, incluyendo características propias de la población infantil. Algunos de los aspectos tenidos en cuenta son los siguientes:

- Tipo de guardería o escuela y en el caso de que no vaya a la escuela, quien lo cuida

- Listado de trastornos crónicos específicos

- Calidad de vida: Se ha medido con el "KIDSCREEN-10" que capta la multidimensionalidad de la salud ${ }^{23}$. Además se ha incluido la subdimensión de comportamientos de riesgo que pueden influir en la salud individual de la versión española del Child Health and Illness Profile-Adolescent Edition (CHIP-AE) ${ }^{24}$.

- La salud mental: La valoración de los 


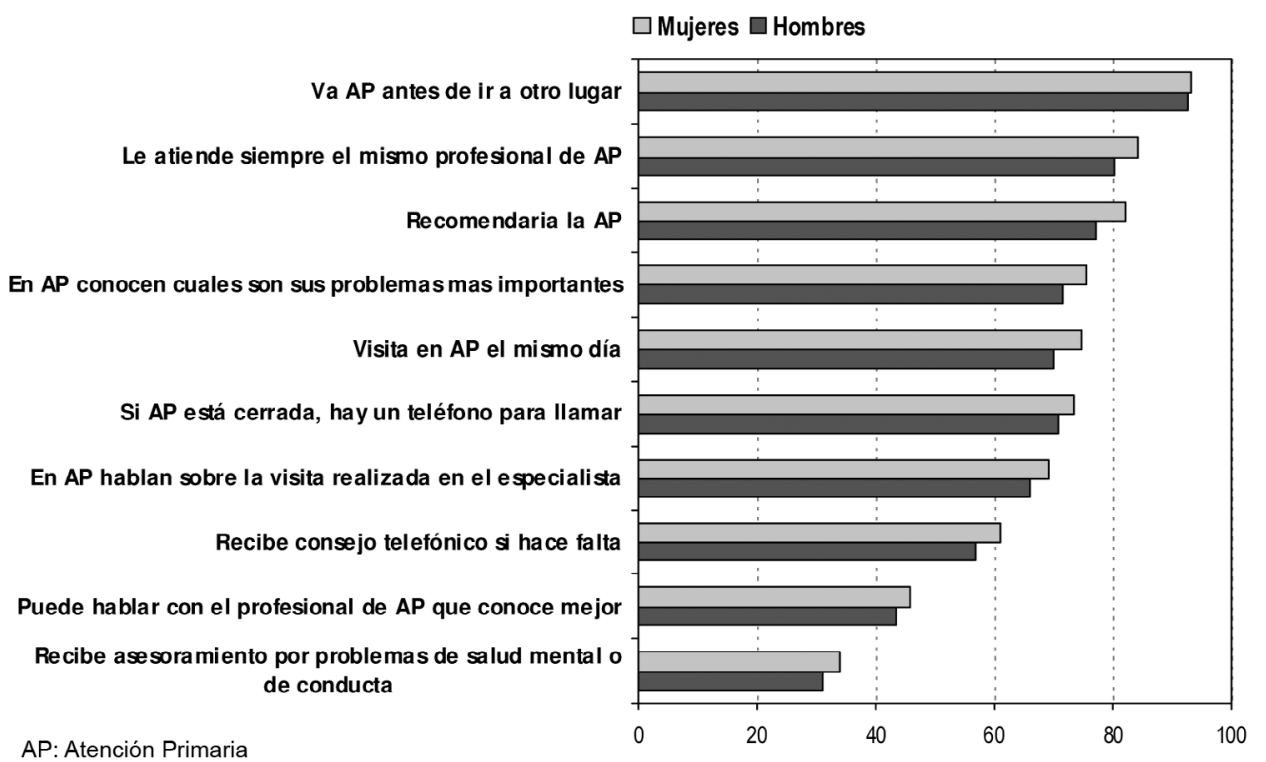

Figura 4 - Características de la Atención Primaria de Salud según sexo. Encuesta de Salud de Barcelona-2006

Figure 4 - Primary healthcare features according to gender. Encuesta de Salud de Barcelona-2006

problemas de salud mental de las criaturas se ha medido con el Strengths and Difficulties Questionnaire (SDQ) ${ }^{25}$, que puntúa cuatro tipos de problemas (emocionales, de conducta, de falta de atención e hiperactividad y problemas con los iguales).

- Actividad física escolar o extra-escolar: La actividad física se mide con la dimensión pertinente del Child Health and Illness Profile-Children Edition (CHIP-CE), diseñado para criaturas de 6 a 11 años.

- Actividades extra-escolares deportivas o no deportivas (música, danza, idiomas...) y otras actividades de ocio como por ejemplo ver la televisión o jugar con ordenadores.

- Lactancia materna e introducción a la dieta.

- Hábitos alimentarios (almuerzo y comida saludable).

- Medidas de sujeción de los menores cuando van en coche.

- Respecto al apartado de utilización de servicios sanitarios cabe destacar el cuestionario PCAT para menores, así como los motivos de salud por los que acude al dentista

\section{Conclusiones}

Las encuestas de salud son un potente instrumento para conocer aspectos relacionados con la salud de la población. Las encuestas realizadas a lo largo de los años deben balancear la comparabilidad entre ellas, algo que implica no cambiar las metodologías utilizadas, con la innovación progresiva. Sin embargo, las nuevas realidades de la población y los nuevos instrumentos deben favorecer el avance. Las encuestas realizadas en la ciudad de Barcelona pueden servir de ejemplo.

\section{Agradecimientos}

Al equipo que trabajó conjuntamente los cuestionarios de la Enquesta de Salut de Catalunya y de la Enquesta de Salut de Barcelona del año 2006: Lucia Baranda, Pilar Brugulat, Antonia Medina, Elisa Séculi y Vicenç Martínez. 


\section{Referências}

1. Health Interview Surveys. De Bruin A, Picavet HSV, Nossikov A (ed.). Towards internacional harmonization of methods and instruments. WHO Regional Publications, European Series number 58, 1996.

2. The European Union Health Monitoring Programme. Eur J Public Health 2003; 13(3); Supplement.

3. Escribá V. Diseño de cuestionarios. En: Rebagliato M, Ruiz I, Arranz M (eds.). Metodología de Investigación en Epidemiologia. Madrid: Diaz de Santos, 1996.

4. Wright EO. Class counts: comparative studies in class análisis. Cambridge University Press; 2000.

5. Krieger N., Williams DR, Moss NE. Measuring social class in US public health research: concepts, methodologies and guidelines. Annu Rev Public Health 1997: 18; 341378.]

6. Grupo SEE y Grupo SEMFyC. Una propuesta de medida de classe social. Aten Primaria 2000; 25: 350-63.

7. Muntaner C, Borrell C, Benach J, Pasarin MI, Fernandez E. The associations of social class and social stratification with patterns of general and mental health in a Spanish population. Int J Epidemiol 2003; 32(6): 950-8.

8. Borrell C, Muntaner C, Benach J, Artazcoz L. Social class and self-reported health status among men and women: what is the role of work organisation, household material standards and household labour? Soc Sci Med 2004; 58(10): 1869-87.

9. Morrell C, Artazcoz L (coordinadoras). $5^{\text {a }}$ Monografía de la SEE. Investigación sobre género y salud. Barcelona: SEE; 2007.

10. Rohlfs I, Borrell C, Artazcoz L, Escribà-Agüir V. The incorporation of gender perspective into health surveys. $J$ Epidemiol Comm Health 61(Suppl. 2): ii20-5.

11. Artazcoz L, Borrell C, Cortès I, Escribà-Agüir V, Cascant L. Occupational epidmiology and work-related inequalities in health: a gender perspective for two complementary approaches to work and health research. J Epidemiol Comm Health 61(Suppl. 2): ii39-45.

12. Solé C. Inmigración interior e inmigración exterior. Papers 2000; 60:211-24.

13. Ribas N. Immigració a Catalunya als anys noranta, quencom de nou? Revista Catalana de Sociología 1997; 6:33-48.

14. Departament d'Estadística. Ajuntament de Barcelona. Disponible en www.ben.es.estadistica [página consultada en Marzo de 2006].
15. Krieger N. Genders, sexes, and health: what are the connections - and why does it matter? Int J Epidemiol 2003; 32(4); 652-7.

16. Krieger N. Discrimination and health. En: Berckman L, Karachi I. Society and health. Oxford: Oxford University Press, 2000. Págs. 36-75.

17. WHO Multi-country Study on Women's Health and Domestic Violence against Women. WHO; 2005. Disponible en http://www.who.int/gender/violence/ who_multicountry_study/en/index.html

18. Blanco P, Ruiz-Jarabo C, Garcia de Vinuesa L, MartinGarcia M. Partner violence and women's health. En: Borrell C, García-Calvente MM, Martí-Boscà V (eds.). Informe SESPAS 2004. La salud pública desde la perspectiva de género y clase social. Gac Sanit 2004; 18(1): 182-8.

19. Krieger N, Smith K, Naishadham D, Hartman C, Barbeau EM. Experiences of discrimination: validity and reliability of a self-report measure for population health research on racism and health. Soc Sci Med 2005; 61 (7): 1576-96.

20. Pantzer K, Rajmil L, Tebe C, Codina F, Serra-Sutton V, Ferrer M, Ravens-Sieberer U, Simeón MC, Alonso J. Health related quality of life in immigrants and native school aged adolescents in Spain. JEpidemiol Community Health 2006: 60(8): 694-8.

21. Pasarín MI, Berrad S, Rajmil L, Solans M, Borrell C, Starfield B. Um instrumento para la evaluación de la atención primaria de salud desde la perspectiva de la población. Aten Primaria 2007; 38: 395-401.

22. Harzheim E, Starfield B, Rajmil L, Alvarez-Dardet C, Stein AT. Internal consistency and reliability of Primary Care Assessment Tool (PCATool-Brasil) for child health services. Cad Saúde Pública 2006; 22(8): 1649-59.

23. Screening for and promotion of health related quality of life in children and adolescents: a European public health perspectiva. Alemania: The KIDSCREEN Group; 2001. Disponible en http://www.kidscreen.org/

24. Rajmil L, Serra-Sutton V, Alonso J, Starfield B, Riley AW, Vázquez JR. The Spanish version of the Chile Health and Illness Profile-Adolescent Edition (CHIP-AE). Qual Life Res 2003; 12; 303-13.

25. Goodman R. The Strenghts and Difficulties Questionnaire: a research note. JChild Psicol. Psychiatry 1997: 38(5): 581-6. 